\title{
$N=2$ Supersymmetric Quantum Mechanics on Riemann Surfaces with Meromorphic Superpotentials
}

\author{
N. V. Borisov ${ }^{1, \star}$, K. N. Ilinski ${ }^{2}$ \\ 1 Institute of Physics, Saint Petersburg University, Ulianovskaya 1, 198904 Saint Petersburg, \\ Russian Federation \\ 2 Saint Petersburg Steklov Mathematical Institute, Fontanka 27, 191011 Saint Petersburg, \\ Russian Federation
}

Received: 29 March 1993/in revised form 6 July 1993

\begin{abstract}
We construct $N=2$ supersymmetric quantum Hamiltonians with meromorphic superpotentials on compact Riemann surfaces and investigate the topological properties of these Hamiltonians. $L_{2}$-cohomology groups for supercharge (a deformed $\bar{\partial}$ operator) are considered and the Witten index for the supersymmetric Hamiltonian with meromorphic superpotential is calculated in terms of Euler characteristic of the Riemann surface and the degree of a divisor of poles for the differential of the superpotential.
\end{abstract}

\section{Introduction}

The conception of supersymmetry was introduced as a theoretical construction in the quantum theory of fundamental interactions [1]. Now this conception has useful applications in quantum mechanics [2] and mathematical physics [3] as a basis for the investigations of topological properties of the Hamiltonians and elliptic complexes. Supersymmetric scattering theory [4] gives a general approach to generalizations of index theorems on elliptic operators with continuous spectrum and to the investigation of topological properties of scattering matrices.

It is especially important to investigate the supersymmetric Hamiltonian $H$ with nontrivial Witten index $\Delta_{W}(H)$ [or supersymmetric scattering index $n\left(H, H_{0}\right)$ ]. The classical Hodge-de Rham theory states that the Witten index for the Laplace operator on forms on the compact Riemannian manifold with smooth metric is equal to the Euler characteristic of the manifold. Another example of the supersymmetric Hamiltonian with nontrivial Witten index arises in the holomorphic $N=2$ WessZumino model in supersymmetric quantum mechanics which was introduced in $[5,6]$ and developed in [7-9]. It was discovered in [5] for the supersymmetric Hamiltonian on a (noncompact) complex plane with polynomial superpotential $f$ the Witten index

\footnotetext{
* This work was supported, in part, by a Soros Foundation Grant awarded by the American Physical Society
} 
is connected with degree of polynomial $\left(\Delta_{W}(H)=\operatorname{deg}(f)-1\right)$. On the Riemann sphere the polynomial corresponds to the meromorphic function with a pole at infinity. These exmples show the importance of the topology of manifolds and singularities of superpotentials for the value of Witten indexes. That is why it is interesting to investigate the supersymmetric Hamiltonian with general meromorphic superpotential on the compact Riemann surface of arbitrary genus. The problem is considered in this paper.

In the paper [8] the case of general meromorphic superpotentials $f$ on the complex plane with euclidean metric was treated. The paper contains a mathematically rigorous approach to the definitions of self-adjoint extensions of supercharges. It was shown that for a general meromorphic superpotential there are two different self-adjoint extensions of the supercharges which are non-Fredholm operators. In the particular case of $f(z)=\lambda z^{-p}(p \in N, \lambda \in C \backslash\{0\})$ these two supercharges coincide and the Witten index is equal to $1-p$. The mathematical problems in the case of meromorphic superpotentials on the complex plane with euclidean metric are due to the finite volume of compact neighbourhoods of poles of the superpotential. This point crucially differs this case from the case of the polynomial superpotential which increases at euclidean infinity. In our approach we propose to consider the metrics on Riemann surfaces which are euclidean at infinity at the poles of superpotential. (The supersymmetric scattering theory for the supersymmetric Hamiltonian on the Riemannian spaces with an euclidean at infinity metric and zero superpotential was constructed in [4].) In such a metric in our case we will have the compactness of the resolvent of the supersymmetric Hamiltonian and Fredholmness of supercharges.

On the other hand in [10] the attention was attracted to the investigations of $L_{2}$-cohomologies of the complex which arises in $N=2$ Wess-Zumino quantum mechanics. Motivated by their work in [9] cohomology groups for the perturbed Dolbeault operator were considered and their connection with cohomologies of Koszul complex was discovered. $N=2$ supersymmetric quantum mechanics on $C^{n}$ with the holomorphic superpotential was considered in [9]. It was shown that zero-modes of the supersymmetric Hamiltonian lie in the subspace of differential forms of middle degree and the number of zero-modes is equal to the number of zeros of the differential of the holomorphic superpotential.

In [9] the connections between smooth cohomologies of the operator $\bar{\partial}_{f}=\bar{\partial}+f$, $f$-holomorphic one-form, and local rings of singularities for the general case of Stein spaces were investigated. To understand the direct connection between the $L_{2}$ cohomologies of the supercharge and the structure of singularities of the superpotential in the general case it is important to investigate $N=2$ supersymmetric quantum mechanics with arbitrary meromorphic superpotential on a general compact Riemann surface.

In this paper we construct an $N=2$ supersymmetric Hamiltonian with meromorphic superpotential on arbitrary genus Riemann surface as on the Riemannian space which is euclidean at infinity in pole points of the superpotential. We prove the analog of the Hodge Theorem for our complex and calculate the Witten index for this supersymmetric Hamiltonian.

The paper is organized as follows. In Sect. 2 we construct supersymmetric quantum mechanics with meromorphic superpotential on Riemann surfaces which we consider as a euclidean at infinity manifolds. In Sect. 3 we describe the corresponding $L_{2}$-cohomology and we prove the analog of the Hodge Theorem. In Sect. 4 we calculate the Witten index for the supersymmetric Hamiltonian with meromorphic superpotential and in Sect. 5 we formulate a conclusion about the necessity of the 
requirement of euclidean at infinity metric and we reformulate the result for the Witten index in terms of the Euler characteristic of compact Riemann surfaces and the divisor of poles for the differential of superpotential.

\section{2. $N=2$ Supersymmetric Quantum Mechanics with Meromorphic Superpotentials on Riemann Surfaces}

The algebra of $N=2$ supersymmetric quantum mechanics

$$
H=Q^{+} Q^{-}+Q^{-} Q^{+}, \quad\left(Q^{+}\right)^{2}=0, \quad\left(Q^{-}\right)^{2}=0, \quad\left(Q^{+}\right)^{*}=Q^{-}
$$

has an algebraically equivalent form for self-adjoint supercharges: $Q_{1}=Q^{+}+Q^{-}$ and $Q_{2}=i\left(Q^{+}-Q^{-}\right)$,

$$
\begin{gathered}
H=Q_{1}^{2}=Q_{2}^{2}, \quad\left\{Q_{1}, Q_{2}\right\}=Q_{1} Q_{2}+Q_{2} Q_{1}=0, \\
Q_{1}^{*}=Q_{1}, \quad Q_{2}^{*}=Q_{2}
\end{gathered}
$$

For unbounded operators in Hilbert space $\mathbf{H}$ we suppose that these relations take place on dense $Q^{ \pm}$-invariant domain $D$, where the Hamiltonian $H$ is essentially self-adjoint.

If we are interested in the Witten index we need to fix, in addition to (1) or (2), unitary self-adjoint involution $\tau\left(\tau^{*}=\tau^{-1}=\tau\right)$ on $\mathbf{H}$ such that

$$
\left\{\tau, Q^{+}\right\}=\left\{\tau, Q^{-}\right\}=0 \quad \text { or } \quad\left\{\tau, Q_{1}\right\}=\left\{\tau, Q_{2}\right\}=0 .
$$

The Witten index for supersymmetric Hamiltonian $H$ relative to involution $\tau$ is defined as [2]

$$
\Delta_{W}(H, \tau)=\operatorname{dim} P_{+} \mathbf{H}(0)-\operatorname{dim} P_{-} \mathbf{H}(0),
$$

where $P_{ \pm}$-spectral projection on the eigenspace of $\tau$ with eigenvalue \pm 1 and $\mathbf{H}(0)$ is the eigensubspace of $H$ corresponding to zero eigenvalue.

To construct the $N=2$ supersymmetric Hamiltonian with meromorphic superpotential with poles in $z_{1}, \ldots, z_{n}$ on the arbitrary genus compact Riemann surface $M_{0}$ we need to introduce on the manifold $M=M_{0} \backslash\left\{z_{1}, \ldots, z_{n}\right\}$ a Riemannian metric $g$ which is euclidean at infinity in the points $z_{1}, \ldots, z_{n}$. This means that there are open neighborhoods $O_{R_{i}}$ of $z_{i}$ and diffeomorphic maps $\phi_{i}$ of $O_{R_{2}} \backslash\left\{z_{i}\right\}$ to open sets $C B_{R_{\imath}}=\left\{u \in C:|u|>R_{\imath}\right\}$ on complex plane such that on each $O_{R_{i}}$ the metric is the pullback by $\phi_{\imath}$ of the euclidean metric on $C B_{R_{\imath}}$. Besides this everywhere below the metric $g$ will be assumed to be a Kähler one. It is possible to construct such a metric starting from the arbitrary Kähler metric on the compact Riemann surface $M_{0}$ by means of sticking together this metric on $M \backslash \cup_{i} O_{R_{\imath}}$ with the euclidean ones in the vicinities $O_{R_{r}}$ of $z_{\imath}$.

For the meromorphic superpotential $f(z)$ on $M_{0}$ with the poles in points $\left\{z_{1}, \ldots, z_{n}\right\} \in M_{0}$ we define supercharge $Q_{c}^{+}$on smooth differential forms with compact support $\Lambda_{c}(M) \equiv \bigoplus_{k=0}^{2} \Lambda_{c}^{k}(M), M=M_{0} \backslash\left\{z_{1}, \ldots, z_{n}\right\}$ as the deformed Dolbeault operator

$$
Q_{c}^{+}=\bar{\partial}_{V}=\bar{\partial}+V
$$

where $\bar{\partial}=\frac{\partial}{\partial \bar{z}} d \bar{z} \wedge$ and $V=\frac{\partial f(z)}{\partial z} d z \wedge$. 
The adjoint supercharge $Q_{c}^{-}$and symmetrical supercharge $Q_{c}$ are defined as

$$
Q_{c}^{-}=\left.\left(Q_{c}^{+}\right)^{*}\right|_{\Lambda_{c}(M)}, \quad Q_{c}=Q_{c}^{+}+Q_{c}^{-} .
$$

The hermitian conjugation (h.c.) is considered here relative to the scalar product on $\Lambda_{c}(M)$, defined by the metric $g$ on $M$ :

$$
(\omega, \phi)=\int_{M} \omega \wedge * \phi \equiv \int_{M}\langle\omega \mid \phi\rangle,
$$

where $*$ is the Hodge star operator. The operators $Q_{c}^{+}, Q_{c}^{-}$and $H_{c}=Q_{c}^{+} Q_{c}^{-}+Q_{c}^{-} Q_{c}^{+}$ give the realization of the algebra of $N=2$ supersymmetric quantum mechanics on $\Lambda_{c}(M)$.

Let us start with the following lemma:

Lemma 2.1. The supercharge $Q_{c}^{+}$is closable operator in Hilbert space $\mathbf{H} \equiv \Lambda_{2}(M)=$ $\Lambda_{c}(M)$ of square integrable forms on $M$ and

$$
\overline{Q_{c}^{+}} \cdot \overline{Q_{c}^{+}}=0 \text {. }
$$

Proof. If $x_{n} \in \Lambda_{c}(M), x_{n} \rightarrow 0$, and $Q_{c}^{+} x_{n} \rightarrow y$ then for arbitrary $\omega \in \Lambda_{c}(M)$,

$$
(y, \omega)=\lim _{n \rightarrow \infty}\left(Q_{c}^{+} x_{n}, \omega\right)=\left(x_{n},\left(Q_{c}^{+}\right)^{*} \omega\right)=0,
$$

i.e. $y=0$ due to the density of $\Lambda_{c}(M)$ in $\Lambda_{2}(M)$.

To prove (6) we represent arbitrary $x \in D\left(\overline{Q_{c}^{+2}}\right)=\left\{x \in D\left(\overline{Q_{c}^{+}}\right): \overline{Q_{c}^{+}} x \in D\left(Q_{c}^{+}\right)\right\}$ by $x_{n} \in \Lambda_{c}(M)$, such that $x_{n} \rightarrow x$ and $Q_{c}^{+} x_{n} \rightarrow \overline{Q_{c}^{+}} x$.

Due to $Q_{c}^{+}\left(Q_{c}^{+}\right) x_{n}=\left(Q_{c}^{+}\right)^{2} x_{n}=0$ and closeness of $\overline{Q_{c}^{+}}$we have $\overline{Q_{c}^{+}} x \in D\left(\overline{Q_{c}^{+}}\right)$ and $\overline{Q_{c}^{+}}\left(\overline{Q_{c}^{+}} x\right)=0$.

Let us define supercharges $Q^{+}, Q^{-}, Q$,

$$
Q^{+} \equiv \overline{Q_{c}^{+}}, \quad Q^{-} \equiv\left(\overline{Q_{c}^{+}}\right)^{*}=\left(Q^{+}\right)^{*}, \quad Q \equiv Q^{+}+Q^{-} .
$$

Supercharges $Q^{+}, Q^{-}$are closed operators with dense domains of definition $D\left(Q^{ \pm}\right)$, $\Lambda_{c}(M) \subset D\left(Q^{ \pm}\right)$, and $\left(Q^{ \pm}\right)^{2}=0$,

Lemma 2.2. $Q_{c}$ and $H_{c}=Q_{c}^{2}$ are essentially self-adjoint operators and

$$
\overline{Q_{c}}=Q, \quad H \equiv \overline{H_{c}}=Q^{2} .
$$

Proof. First of all due to a Glimm-Jaffe-Nelson type theorem on essentially selfadjointness of symmetric operators [16], if $Q_{c}^{2}$ is an essentially self-adjoint operator, then $Q_{c}$ is also an essentially self-adjoint one. That is why it is sufficient to prove the essentially self-adjointness of the Hamiltonian $H_{c}=Q_{c}^{2}$ on $\Lambda_{c}(M)$. In the paper [11] the general methods for the proof of self-adjointness of the square of general Dirac operators on complete Riemannian manifolds were developed. Due to our choice of metric $g$ on $M=M_{0} \backslash\left\{z_{1}, \ldots, z_{n}\right\}$, which is euclidean at infinity in each $z_{\imath}$, $i=1, . \quad, n$, our manifold $(M, g)$ is complete. That is why we can apply general methods from [11] to our case.

Now we have to show that self-adjoint operator $\overline{Q_{c}}$ coincides with the operator $Q$. To see this let us construct by means of the operator $Q$ the sesquilinear form $a(\cdot, \cdot)$ on the domain $D[a]=D\left(Q^{+}\right) \cap D\left(Q^{-}\right)$defined as

$$
a(x, y)=(Q x, Q y), \quad x, y \in D[a] .
$$


Then we have due to Lemma 2.1 that $a(x, y)=\left(Q^{+} x, Q^{+} y\right)+\left(Q^{-} x, Q^{-} y\right)$ and that sesquilinear form $a(\cdot, \cdot)$ is a non-negative closed semi-bounded form. The standard representation theorem for closed, semi-bounded sesquilinear forms [16] immediately gives us that there exists a unique non-negative self-adjoint operator $\tilde{H}$ such that $D\left(\tilde{H}^{1 / 2}\right)=D[a]$ and

$$
\left(\tilde{H}^{1 / 2} x, \tilde{H}^{1 / 2} y\right)=a(x, y) \quad \text { for } \quad x, y \in D\left(\tilde{H}^{1 / 2}\right) .
$$

The operator $\tilde{H}$ coincides with $H$ because $\left.\tilde{H}\right|_{\Lambda_{c}(M)}=H_{c}$ and $H=\overline{H_{c}}$. So the form $a(\cdot, \cdot)$ corresponds to the operator $H=\bar{Q}_{c}^{2}$ and we can consider the operator $\tilde{H}^{1 / 2}=\bar{Q}_{c}$. Then

$$
\left.\left(Q=Q_{c}\right)\right|_{\operatorname{Ker} H}=0 \quad \text { and }\left.\quad\left(Q=U \bar{Q}_{c}\right)\right|_{(\operatorname{Ker} H)^{\perp} \cap D(Q)},
$$

where $U$ is an isometric operator. Using the equality $\left.Q\right|_{\Lambda_{c}(M)}=Q_{c}$ we obtain $U=I$ and $\overline{Q_{c}}=Q$.

Remark. As we saw from the proof of Lemma 2.2 the essentially self-adjointness of the Hamiltonian $H_{c}=Q_{c}^{2}$ on $\Lambda_{c}(M)$ is closely related to our choice of Riemannian metric $g$, which is euclidean at infinities in the poles $\left\{z_{1}, \ldots, z_{n}\right\}$ of the meromorphic superpotential $f(z)$. We will have different results if we consider the self-adjoint extensions of $Q_{c}$ and $H_{c}=Q_{c}^{2}$ defined on $\Lambda_{c}(M), M=M_{0} \backslash\left\{z_{1}, \ldots, z_{n}\right\}$ in the case of compact Riemann surface $M_{0}$ with smooth metric $g_{0}$. The careful analysis for the case of the Riemann sphere with ordinary euclidean metric and meromorphic superpotential was developed in [8] and gave the description of two different types of self-adjoint extensions of $Q_{c}$ and sufficient conditions for their coincidence. (We are grateful to the referee for the information about the paper [8].)

\section{3. $L_{2}$-Cohomology of Deformed Dolbeault Operator and Zero-Modes of $N=2$ Supersymmetric Hamiltonian}

In this section we construct the analog of the Hodge theorem for the differential complex, generated by the closed deformed Dolbeault operator $Q^{+}=(\bar{\partial}+V)$ in the space $\Lambda_{2}(M) \equiv \bigoplus_{k=0}^{2} \Lambda_{2}^{k}(M), M=M_{0} \backslash\left\{z_{1}, \ldots, z_{n}\right\}$, where $M_{0}$ is a compact Riemann surface. Noncompact Riemann surface $M$ is a 1-dimension complex Stein space [12] and for the unperturbed Dolbeault operator $\vec{\partial}$ on the space of smooth forms,

$$
\Lambda(M)=\bigoplus_{k=0}^{2} \Lambda^{k}(M)=\bigoplus_{k=0}^{2} \bigoplus_{p+q=k, p, q>0} \Lambda^{p, q}(M),
$$

the smooth cohomology groups are [12]:

$$
H_{\bar{\partial}}^{p, q}(M)=\left.\operatorname{Ker} \bar{\partial}\right|_{\Lambda^{p, q}(M)} / R\left(\left.\bar{\partial}\right|_{\Lambda^{p, q-1}(M)}\right)=\left\{\begin{array}{ll}
0 & q=1 \\
\Omega^{p}(M) & q=0
\end{array},\right.
$$

where $\Omega^{p}(M)$ is the space of holomorphic $p$-forms on $M$.

For perturbed Dolbeault operator $Q^{+}=(\bar{\partial}+V)$ on the space of all smooth forms $\Lambda(M)$ the cohomology groups for the complex

$$
\begin{gathered}
\Lambda^{0}(M) \stackrel{Q^{+}}{\longrightarrow} \Lambda^{1}(M) \stackrel{Q^{+}}{\longrightarrow} \Lambda^{2}(M), \\
H^{k}(M)=\left.\operatorname{Ker}(\bar{\partial}+V)\right|_{\Lambda^{k}(M)} /\left.R(\bar{\partial}+V)\right|_{\Lambda^{k-1}(M)}, \quad k=0,1,2
\end{gathered}
$$


are isomorphic to the cohomology groups of the Koszul complex

$$
\begin{gathered}
\Omega^{0}(M) \stackrel{V}{\longrightarrow} \Omega^{1}(M), \\
K_{V}^{p}(M)=\left.\operatorname{Ker} V\right|_{\Omega^{p}(M)} / R\left(\left.V\right|_{\Omega^{p-1}(M)}\right), \quad p=0,1,
\end{gathered}
$$

and

$$
K_{V}^{p}(M)=\left\{\begin{array}{ll}
0 & p=0 \\
\oplus R_{z_{\imath}}^{f} & p=1
\end{array},\right.
$$

where $R_{z}^{f} \equiv O_{z} /[\partial f]_{z}, O_{z}$ denotes the ring of germs of holomorphic functions in the vicinity of $z$ and $[\partial f]_{z}$ is the ideal in $O_{z}$, generated by the $\frac{\partial f}{\partial z}[9]$.

We introduce $L_{2}$-cohomology groups for the supercharge $Q^{+}=(\bar{\partial}+V)$ in Hilbert space of square integrable forms $\Lambda_{2}(M)=\bigoplus_{k=0}^{2} \Lambda_{2}^{k}(M)$,

$$
H_{2, V}^{k}(M)=\left.\operatorname{Ker}(\bar{\partial}+V)\right|_{\Lambda_{2}^{k}(M)} /\left.R(\bar{\partial}+V)\right|_{\Lambda_{2}^{k-1}(M)}
$$

and investigate the connections of these groups with the spaces of zero-modes of our $N=2$ supersymmetric Hamiltonian (perturbed Laplace operator)

$$
H=\bar{\Delta}(V)=\left((\bar{\partial}+V)(\bar{\partial}+V)^{*}+(\bar{\partial}+V)^{*}(\bar{\partial}+V)\right) .
$$

We start with two propositions relative to the operator $\bar{\Delta}(V)$ :

Lemma 3.1. 1. Operator $\bar{\Delta}(V)$ has a form

$$
H=\bar{\Delta}(V)=H_{0}+\bar{b}^{*} b \bar{\partial} \overline{\left(\frac{1}{\alpha} \partial f\right)}+b^{*} \bar{b} \partial\left(\frac{1}{\alpha} \partial f\right) \equiv H_{0}+K,
$$

where $H_{0}=\frac{1}{2} \Delta+\frac{2}{\alpha}|\partial f|^{2}, \Delta=2\left\{\bar{\partial}, \bar{\partial}^{*}\right\}$ is the usual Laplace operator on Riemannian manifold $M$ with Kähler metric $g, \alpha=g_{z \bar{z}}, b^{*} \equiv d \bar{z} \wedge, b^{*} \equiv d z \wedge$, and $\bar{b}, b$ are adjoint operators for $\bar{b}^{*}, b^{*}$. The operators $\bar{b}^{*}, b^{*}, \bar{b}, b$ obey the following algebra:

$$
\begin{gathered}
\{\bar{b}, \bar{b}\}=\left\{b^{*}, b\right\}=\frac{2}{\alpha}, \quad\left\{\bar{b}^{*}, b\right\}=\left\{b^{*}, \bar{b}\right\}=0, \\
\left(\bar{b}^{*}\right)^{2}=\left(b^{*}\right)^{2}=(\bar{b})^{2}=(b)^{2}=0 .
\end{gathered}
$$

2. For any $\varepsilon>0$ there exists constant $C>0$ such that for any $\omega \in D\left(H_{0}\right)$,

$$
|(K \omega, \omega)| \leq \varepsilon\left(H_{0} \omega, \omega\right)+C(\omega, \omega) .
$$

Proof The first part of this proposition can be proved by direct calculations. To prove the second part of the proposition it is necessary to take into account that under our conditions the operators $\bar{b}^{*}, b^{*}, \bar{b}, b$ are bounded and for any $\varepsilon>0$ there exists the constant $C_{\varepsilon}>0$ such that

$$
\left|\partial^{2} f\right| \leq \varepsilon|\partial f|^{2}+C_{\varepsilon}
$$

for all $z$ on $M$.

Lemma 3.2. Operator $\bar{\Delta}(V)$ has a compact resolvent. 
Proof First of all we prove that the operator $H_{0}$ has a compact resolvent. After this we can use Lemma 3.1 and standard perturbation theory of Kato [14] to prove the statement of this lemma.

The proof of the compactness of the resolvent of $H_{0}$ can be fulfilled in close analogy with the corresponding statement for the usual Schrödinger operator with increasing potential [16]. According to minimax principle it is enough to prove that minimax principle numbers:

$$
\mu_{n}\left(H_{0}\right)=\sup _{\phi_{1}, \quad \phi_{n-1}} \inf _{\substack{\psi \in D\left(H_{0}\right) ;\|\psi\|=1 \\ \psi \in\left[\phi_{1}, \quad, \phi_{n-1}\right]^{\perp}}}\left(\psi, H_{0} \psi\right)
$$

tend to infinity $\left(\mu_{n}\left(H_{0}\right) \rightarrow \infty\right)$ if $n \rightarrow \infty$. For any $B \geq 0$ there exists the set of positive numbers $\left\{R_{1}, \ldots, R_{n}\right\}$ such that $\frac{2}{\alpha}|\partial f|^{2} \geq B$ if $z \in \cup_{i} O_{R_{i}}$. Such a set exists because the function $f$ has poles in the points $\left\{z_{1}, \ldots, z_{n}\right\}$ into the open neighborhoods $O_{R_{\imath}}$. Let us consider the potential $W$ such that

$$
W= \begin{cases}0 & (x, y) \in \cup_{\imath} O_{R_{\imath}} \\ -B & (x, y) \in M \backslash \cup_{\imath} O_{R_{\imath}} .\end{cases}
$$

Then $\frac{2}{\alpha}|\partial f|^{2} \geq B+W$ and $\mu_{n}\left(H_{0}\right) \geq B+\mu_{n}\left(\frac{1}{2} \Delta+W\right)$. Due to $W$ is the potential with compact support $\exists N$ such that $\mu_{n}\left(\frac{1}{2} \Delta+W\right) \geq-1$ for $n \geq N$. Hence $\mu_{n} \geq B-1$ for $n \geq N$ and $\mu_{n}\left(H_{0}\right) \rightarrow \infty$ if $n \rightarrow \infty$ because the parameter $B$ is arbitrary one. So the operator $H_{0}$ has a compact resolvent.

Let us introduce the spaces of zero-modes of the perturbed Laplace operator:

$$
\mathbf{H}^{k}(\bar{\Delta}(V))=\left\{\omega \in D(\bar{\Delta}(V)) \cap \Lambda_{2}^{k}: \bar{\Delta}(V) \omega=0\right\},
$$

then it is possible to formulate the analog of the Hodge Theorem [13]:

Theorem 3.3. 1. $\operatorname{dim} \mathbf{H}^{k}(\bar{\Delta}(V))<\infty$.

2. There is a self-adjoint compact operator $G$ on $\Lambda_{2}(M)$ such that

$$
\Lambda_{2}(M)=\operatorname{Ker}(\bar{\Delta}(V)) \oplus Q^{+}\left(Q^{-} G \Lambda_{2}(M)\right) \oplus Q^{-} G\left(Q^{+} \Lambda_{2}(M)\right) .
$$

Proof The inequality $\operatorname{dim} \mathbf{H}^{k}(\bar{\Delta}(V))<\infty$ follows from the compactness of the resolvent of $\bar{\Delta}(V)$ by the standard way.

It is easy to see that the operator $G$ defined as

$$
G=\left(\left.(\bar{\Delta}(V))^{-1}\right|_{(\operatorname{Ker}(\bar{\Delta}(V)))^{\perp}}\right) \circ(I-P),
$$

where $P$ is an orthogonal projection on $\operatorname{Ker}(\bar{\Delta}(V))$, is a self-adjoint positive compact operator in Hilbert space $\Lambda_{2}(M)$. There is the decomposition

$$
\Lambda_{2}(M)=\operatorname{Ker}(\bar{\Delta}(V)) \oplus \overline{R(\bar{\Delta}(V))} .
$$

Using Lemma 2.2 we have

$$
\bar{\Delta}(V)=Q^{-} Q^{+}+Q^{+} Q^{-}, \quad Q^{+2}=Q^{-2}=0, \quad \text { and } \quad\left(Q^{+}\right)^{*}=Q^{-} .
$$

The last equalities show us that $R\left(Q^{+}\right) \perp R\left(Q^{-}\right)$and that

$$
\overline{R(\bar{\Delta}(V))}=R(\bar{\Delta}(V))=R\left(Q^{-} Q^{+}\right) \oplus R\left(Q^{+} Q^{-}\right) .
$$


Then we can infer that

$$
\Lambda_{2}(M)=\operatorname{Ker}(\bar{\Delta}(V)) \oplus R\left(Q^{-} Q^{+}\right) \oplus R\left(Q^{+} Q^{-}\right),
$$

and for all $\omega \in \Lambda_{2}(M)$

$$
\omega=P \omega+Q^{+} Q^{-} G \omega+Q^{-} Q^{+} G \omega .
$$

Following the proof of this fact for the usual Laplace operator [13] it is not difficult to show that for any operator $T$ such that

$$
T \bar{\Delta}(V)=\bar{\Delta}(V) T \quad \text { and } \quad T: D(\bar{\Delta}(V)) \rightarrow D(\bar{D}(V))
$$

the following equality takes place:

$$
T G=G T .
$$

From this fact and the decomposition (9) we immediately have for all $\omega \in \Lambda_{2}(M)$,

$$
\omega=P \omega+Q^{-} G Q^{+} \omega+Q^{+} Q^{-} G \omega .
$$

This equality finishes the proof of the theorem.

\section{Calculation of the Witten Index for Supersymmetric Hamiltonians with Meromorphic Superpotentials on Riemann Surfaces}

For the calculation of the Witten index we shall use the approach developed in [15] for the proof of Morse inequalities for smooth functions on Riemannian manifolds. In this sense our result can be interpreted as the analog of the Morse equality for meromorphic functions.

On the basis of Sect. 2 we conclude that

$$
\Lambda_{2}(M)=\Lambda_{2}^{+}(M) \oplus \Lambda_{2}^{-}(M),
$$

where $\left.\tau\right|_{\Lambda_{2}^{ \pm}(M)}= \pm I$ and

$$
Q=\left(\begin{array}{cc}
0 & A \\
A^{*} & 0
\end{array}\right), \quad H=Q^{2}=\left(\begin{array}{cc}
A A^{*} & 0 \\
0 & A^{*} A
\end{array}\right),
$$

with $A: D(A) \rightarrow \Lambda_{2}^{-}(M)$ - densely defined closed operator on the domain $D(A) \subset$ $\Lambda_{2}^{+}(M)$, where $D(A)=D(Q) \cap \Lambda_{2}^{+}(M)$. From the definition of the Witten index relative to the involution $\tau=(-1)^{N}, N$ is the operator of the order of differential forms

$$
\Delta_{W}(H)=\operatorname{dim}\left(\operatorname{Ker} H \cap \Lambda_{2}^{+}(M)\right)-\operatorname{dim}\left(\operatorname{Ker} H \cap \Lambda_{2}^{+}(M)\right),
$$

we see that

$$
\Delta_{W}(H)=\operatorname{dim} \operatorname{Ker} A^{*}-\operatorname{dim} \operatorname{Ker} A=-\operatorname{ind}(A) .
$$

Lemma 4.1. If we introduce $t \in[1,+\infty)$ as a deformation parameter in the definitions of supercharges by means of the substitution $t V$ instead of $V$ in (4), then $\operatorname{ind}\left(A_{t}\right)$ is independent from $t$.

Proof. This fact is a cosequence of the continuity of the map:

$$
\forall T \quad t \in[1, T] t \rightarrow Q(t)=\bar{\partial}+t \partial f b^{*}+\text { h.c. } \in \operatorname{DIR}\left(\Lambda_{2}(M)\right),
$$

where $\operatorname{DIR}\left(\Lambda_{2}(M)\right)$ is the space of the operators on $\Lambda_{2}(M)$ which are 
1. odd with respect to the grading $\tau$;

2. self-adjoint;

3. have a compact resolvent

with the following metric:

$$
d\left(Q_{1}, Q_{2}\right)=\left\|\left(Q_{1}+i\right)^{-1}-\left(Q_{2}+i\right)^{-1}\right\| \quad \text { for } \quad Q_{1}, Q_{2} \in \operatorname{DIR}\left(\Lambda_{2}(M)\right) .
$$

This continuity can be proved with a method patterned after [6] using the resolvent identity and the boundedness of $(Q(t)+i)^{-1}\left(\partial f b^{*}+\right.$ h.c. $)\left(Q\left(t^{\prime}\right)+i\right)^{-1}$. On the other hand $\operatorname{ind}(A)$ is a continuous function of $A \in \operatorname{DIR}\left(\Lambda_{2}(M)\right)$ in the described topology [6].

Let us return to the supersymmetric Hamiltonian. According to Lemma 3.1 the operator $H(t)$ has the form:

$$
H(t)=H_{0}(t)+t K .
$$

In our case $K$ is an unbounded operator in $\Lambda_{2}(M)$. This is the main point of the technical difference from the Witten case [15], where $K$ was a bounded operator. Lemma 3.1 gives a strong domination of $H_{0}(t)=\frac{1}{2} \Delta+t^{2} \frac{2}{\alpha}|\partial f|^{2}$ in comparison with $K$. Using this domination we can generalize the Witten approach.

In the operator $H_{0}=\frac{1}{2} \Delta+t^{2} \frac{2}{\alpha}|\partial f|^{2}$ the positive potential $t^{2} \frac{2}{\alpha}|\partial f|^{2}$ tends to infinity: $t|\partial f| \rightarrow \infty$ for $t \rightarrow \infty$ in all points except the critical points of superpotential $f(z)$. Due to the domination of $H_{0}(t)$ it is natural to expect the tendency of the concentration of eigenfunctions of $H(t)$ around the critical points of $f(z)$. The next Lemma 4.2 formalizes this consideration:

Lemma 4.2. Let $N$ be the closed subset in $M$ without critical points of the superpotential $f(z)$, then there exists $C>0$, depending from $N$ and positive real parameter $A$ such that for every eigenfunction $\Phi$ of $H(t)$ with eigenvalue $\lambda \leq A$,

$$
\int_{N}\langle\Phi \mid \Phi\rangle \leq \frac{c}{t}\|\Phi\|_{\Lambda_{2}(M)}^{2} .
$$

Proof. In the notations of Lemma 3.1 we have

$$
\left(H_{0}(t) \Phi, \Phi\right)=\lambda \int_{M}(\langle\Phi \mid \Phi\rangle-t\langle K \Phi \mid \Phi\rangle) .
$$

Using the following inequalities: $\lambda \leq A$ and

$$
t|(K \Phi, \Phi)| \leq \varepsilon\left(H_{0}(t) \Phi, \Phi\right)+C_{1}\|\Phi\|_{\Lambda_{2}(M)}^{2}, \quad 1>\varepsilon>0, C_{1}>0,
$$

and reducing the domain of the integration on the left-hand side from $M$ to $N$ one obtains the inequality:

$$
\frac{1-\varepsilon}{2} \int_{N}\left(\frac{4 t^{2}}{\alpha}|\partial f|^{2}\langle\Phi \mid \Phi\rangle\right) \leq\left(A+C_{1}\right)\|\Phi\|_{\Lambda_{2}(M)}^{2} .
$$

According to the definition of $N$ there exists a real positive number $\omega$ such that

$$
\frac{4}{\alpha}|\partial f|^{2} \geq \omega
$$


on $N$. Then from (10) and (11) we obtain the required inequality:

$$
\int_{N}\langle\Phi \mid \Phi\rangle \leq \frac{2\left(A+C_{1}\right)}{(1-\varepsilon) t^{2} \omega}\|\Phi\|_{\Lambda_{2}(M)}^{2}
$$

taking into account that $C_{1}$ in (12) linear depends on $t$.

Theorem 4.3. Witten index for supersymmetric Hamiltonian $H=\bar{\Delta}(V)$ equals to the number of critical points of superpotential $f(z)$ (accounting in according to the degrees of their degeneracy) with sign minus.

Proof. Due to the stability of the Witten index of $\bar{\Delta}(t V)$ relative to the deformation of the positive parameter $t$ it is possible to consider $\Delta_{W}(\bar{\Delta}(t V))$ for $t \rightarrow \infty$. In this case all eigenfunctions of the operator $\bar{\Delta}(t V)$ concentrate in the neighborhoods of critical points of $f(z): z_{i}^{0}, i=1, \ldots, m$, and it is possible to calculate the number of zero-modes. On the first stage we suppose that $f^{\prime}(z)$ has only nondegenerated zeros and the metric $g$ is euclidean in the neighborhoods of these zeros. We overcome these conditions at the end of our proof.

First of all we shall prove that $\bar{\Delta}(t V)$ has at least $m$ eigenvalues which increase slower than $t$ for $t \rightarrow \infty$ with the eigenfunctions in $\Lambda_{2}^{-}(M)$. Then we show that all eigenvalues of $\bar{\Delta}(t V)$ with eigenfunctions in $\Lambda_{2}^{+}(M)$ and in $\Lambda_{2}^{-}(M)$ (except $m$ previously considered eigenvalues) increase proportional to $t$ for $t \rightarrow \infty$. Due to supersymmetry of $\bar{\Delta}(t V)$ this means in fact that the first $m$ eigenvalues are zero eigenvalues of $\bar{\Delta}(t V)$ with the eigenfunctions in $\Lambda_{2}^{-}$and other non-zero ones.

In order to prove that there exist $m$ eigenvalues slowly dependent from $t$ we use the minimax principle. According to this principle we need to construct $m$ mutually orthogonal vectors $\omega_{1}, \ldots, \omega_{n} \in D(\bar{\Delta}(t V)) \cap \Lambda_{2}^{-}(M)$ such that

$$
\left(\bar{\Delta}(t V) \omega_{i}, \omega_{i}\right) \leq B(t)\left(\omega_{i}, \omega_{i}\right),
$$

where $B(t)=o(t)$. We try to define these functions $\omega_{i}$ as localized ones in the neighborhoods $\Gamma_{\imath}$ of the critical points $z_{i}^{o}$ of the superpotential $f(z)$, where

$$
\partial f(z)=\frac{c_{i}}{2} \hat{z}+\hat{z}^{2} b_{i}(\hat{z}), \quad c_{i} \neq 0, \hat{z}=z-z_{i}^{0} .
$$

Let us introduce smooth functions $\phi_{\varepsilon, i}(\hat{z}, \hat{\bar{z}})$ with compact supports in euclidean neighbourhoods of $z_{i}^{0}$. To construct these functions we use positive $C^{\infty}$-function on $(-\infty, \infty)$ with the following property

$$
\phi(x)= \begin{cases}0 & x \notin]-2,2[ \\ 1 & x \in[-1,1]\end{cases}
$$

and positive number

$$
\varepsilon \leq \min \left\{\tilde{\varepsilon}, \frac{1}{2} \max _{i, j}\left|z_{i}^{0}-z_{j}^{0}\right|\right\}
$$

where $\tilde{\varepsilon}$ is the minimal radius of local euclidean neighborhoods of $z_{i}^{0}$, and we define

$$
\phi_{\varepsilon, i}(\hat{z}, \hat{\bar{z}})=\phi\left(\varepsilon^{-1} 2 \sqrt{2} \operatorname{Re}\left(z-z_{i}^{0}\right)\right) \phi\left(\varepsilon^{-1} 2 \sqrt{2} \operatorname{Im}\left(z-z_{i}^{0}\right)\right) .
$$

Using these notations we introduce $\left\{\omega_{\imath}\right\}_{i=1}^{m}$ as

$$
\omega_{i}=\phi_{\varepsilon, i}(\hat{z}, \hat{\bar{z}}) \exp \left(-\frac{1}{2} t\left|c_{i}\right||\hat{z}|^{2}\right)\left(d z-\frac{\left|c_{i}\right|}{c_{i}} d \bar{z}\right) \text {. }
$$


According to the definition of $\omega_{i}$ they have disjoint supports and hence they are mutually orthogonal for $i \neq j$.

In order to investigate the behaviour of $\left(\bar{\Delta}(t V) \omega_{i}, \omega_{i}\right)$ for $t \rightarrow \infty$ we need to take into account that $\omega_{i}$ concentrate in the euclidean neighbourhoods of $z_{i}^{0}$ and extract the terms of maximum order in $t$. They have the forms

$$
\begin{aligned}
& t^{2} \iint_{-\infty}^{\infty} \frac{d z d \bar{z}}{2 i} \phi_{\varepsilon, i}^{2}(z, \bar{z}) e^{-t\left|c_{\imath}\right||z|^{2}}\left|z^{3} b_{i}(z)\right| \equiv j_{1}(t) \\
& t \iint_{-\infty}^{\infty} \frac{d z d \bar{z}}{2 i} \phi_{\varepsilon, i}^{2}(z, \bar{z}) e^{-t\left|c_{i}\right||z|^{2}}\left|z b_{i}(z)\right| \equiv j_{2}(t)
\end{aligned}
$$

Due to the inequalities

$$
\begin{aligned}
& j_{1}(t) \leq \frac{1}{\sqrt{t}} \max _{[-\varepsilon, \varepsilon] \times[-\varepsilon, \varepsilon]}\left|b_{i}(z)\right| \iint_{-\infty}^{\infty} e^{-\left|c_{i}\right||z|^{2}}|z|^{3} \frac{d z d \bar{z}}{2 i} \\
& j_{2}(t) \leq \frac{1}{\sqrt{t}} \max _{[-\varepsilon, \varepsilon] \times[-\varepsilon, \varepsilon]}\left|b_{i}(z)\right| \iint_{-\infty}^{\infty} e^{-\left|c_{i}\right||z|^{2}}|z| \frac{d z d \bar{z}}{2 i}
\end{aligned}
$$

and

$$
\left(\omega_{i}, \omega_{\imath}\right) \geq \frac{\text { const }}{t} \iint_{-\varepsilon}^{\varepsilon} e^{-\left|c_{i}\right||z|^{2}} \frac{d z d \bar{z}}{2 i}
$$

we have

$$
j_{1}(t)+j_{2}(t) \leq \text { const }^{\prime} \sqrt{t}\left(\omega_{i}, \omega_{i}\right) .
$$

We also have the term

$$
\iint_{-\infty}^{\infty} d^{2} x e^{-\left|c_{\imath}\right||\vec{x}|^{2} t}\left(-\frac{1}{2}\left(\Delta_{\vec{x}} \phi_{\varepsilon, i}\right) \cdot \phi_{\varepsilon, i}+\frac{1}{2}\left|c_{i}\right| t(\vec{x} \vec{\nabla}) \phi_{\varepsilon, i} \cdot \phi_{\varepsilon, i}\right) \equiv j_{3}(t)
$$

and the inequality for this term

$$
j_{3}(t) \leq \text { const } \iint d^{2} x \phi_{\varepsilon, i}^{2} e^{-t\left|c_{\imath}\right||x|^{2}} .
$$

On the basis of these inequalities we obtain

$$
\left(\bar{\Delta}(t V) \omega_{i}, \omega_{i}\right) \leq \mathrm{const} \sqrt{t}\left(\omega_{i}, \omega_{i}\right) .
$$

In order to prove that all eigenvalues of $\bar{\Delta}(t V)$ with eigenfunctions in $\Lambda_{2}^{+}(M)$ and in $\Lambda_{2}^{-}(M)$ (except $m$ previously considered eigenvalues) increase proportional to $t$ for $t \rightarrow \infty$ we use once more the minimax principle. We start with the proof of the following inequality:

$$
(\bar{\Delta}(t V) \omega, \omega) \geq \gamma t(\omega, \omega)
$$

for some $\gamma>0$ and $\forall \omega \in \Lambda_{2}^{+}(M) \cap D(\bar{D}(t V))$. After this we prove the corresponding fact for $\Lambda_{2}^{-}(M)$. 
Let us consider

$$
\begin{aligned}
(\bar{\Delta}(t V) \omega, \omega) & =\int_{M}\left(\left\langle\left(\bar{\partial}+\bar{\partial}^{*}\right) \omega \mid\left(\bar{\partial}+\bar{\partial}^{*}\right) \omega\right\rangle+\left\langle\left(\Delta(t V)-\frac{1}{2} \Delta\right) \omega \mid \omega\right\rangle\right) \\
& \equiv \int_{M} Q(\omega, \omega), \quad \omega \in D(\bar{\Delta}(t V)) .
\end{aligned}
$$

It is possible to divide $M$ on $\cup_{i} \Gamma_{i}$ and $V_{0}=M \backslash \cup_{i} \Gamma_{i}$, where $\left\{\Gamma_{i}\right\}_{\imath=1}^{m}$ are euclidean neighbourhoods of $\left\{z_{i}^{0}\right\}$ considered on the previous step. Hence

$$
(\bar{\Delta}(t V) \omega, \omega)=\int_{V_{0}} Q(\omega, \omega)+\sum_{i} \int_{\Gamma_{i}} Q(\omega, \omega) .
$$

Using Lemma 3.1 and the analog of (7) for $t f$ instead of $f$ it is easy to obtain the following chain of the estimates for the first term:

$$
\begin{aligned}
\int_{V_{0}} Q(\omega, \omega) & \geq \int_{V_{0}}(1-\varepsilon) t^{2}\left\langle\frac{2}{\alpha}|\partial f|^{2} \omega \mid \omega\right\rangle-C_{\varepsilon} t \int_{V_{0}}\langle\omega \mid \omega\rangle \\
& \geq\left((1-\varepsilon) \hat{\varepsilon} t^{2}-C_{\varepsilon} t\right) \int_{V_{0}}\langle\omega \mid \omega\rangle,
\end{aligned}
$$

where

$$
\hat{\varepsilon} \equiv \min _{V_{0}} \frac{2}{\alpha}|\partial f|^{2}
$$

Due to our note at the beginning of the proof we can consider $t>\frac{C_{\varepsilon}+\gamma}{(1-\varepsilon) \hat{\varepsilon}}$ everywhere below. In this case we have the required fact that $\int_{V_{0}} Q(\omega, \omega) \geq \gamma t \int\langle\omega \mid \omega\rangle$.

Now let us consider $\int_{\Gamma_{i}} Q(\omega, \omega), \omega \in \Lambda_{2}^{+}(M) \cap D(\bar{\Delta}(t V))$. It is possible to use the representation of the function $\partial f$ on $\Gamma_{i}$ :

$$
\partial f=\frac{c_{i}}{2} \hat{z}+\hat{z} d_{i}(\hat{z}), \quad \hat{z}=z-z_{i}^{0}, \quad d_{i}(0)=0 .
$$

From the last equality we can infer that there exists $\varepsilon>0$ such that

$$
\frac{c_{\imath}}{2}+d_{i}(\hat{z})>0 \quad \text { for } \quad \hat{z} \in B_{\varepsilon}^{i}=\left\{u \in C:\left|u-z_{\imath}^{0}\right|<\varepsilon\right\}
$$

Then

$$
\int_{\Gamma_{\imath}} Q(\omega, \omega)=\int_{B_{\varepsilon}^{i}} Q(\omega, \omega)+\int_{\Gamma_{i} \backslash B_{\varepsilon}^{i}} Q(\omega, \omega),
$$

and these terms can be estimated as

$$
\begin{gathered}
\int_{\Gamma_{i} \backslash B_{\varepsilon}^{i}} Q(\omega, \omega) \geq t^{2} \min _{\Gamma_{i} \backslash B_{\varepsilon}^{i}}\left(\frac{2}{\alpha}|\partial f|^{2}\right) \int_{\Gamma_{i} \backslash B_{\varepsilon}^{i}}\langle\omega \mid \omega\rangle \\
\int_{B_{\varepsilon}^{i}} Q(\omega, \omega) \geq \int_{B_{\varepsilon}^{i}}\left(\left\langle\frac{\partial}{\partial z} \omega \mid \frac{\partial}{\partial z} \omega\right\rangle+t^{2} \min _{B_{\varepsilon}^{i}}\left(\left|\frac{c_{i}}{2}+d_{i}(\hat{z})\right|^{2}\right)\left\langle|z|^{2} \omega \mid \omega\right\rangle\right) .
\end{gathered}
$$


Now it is necessary to use Lemma A.1 proved in Appendix A:

Lemma A.1. There exists $\gamma(\varepsilon, \beta)>0$ and $t_{0}$ such that for $t>t_{0}$,

$$
\int_{B_{\varepsilon}}\left\{\left|\frac{\partial f}{\partial z}\right|+t^{2} \beta^{2}|z|^{2}|f|^{2}\right\} \frac{d z d \bar{z}}{2 i} \geq \gamma(\varepsilon, \beta) t \int_{B_{\varepsilon}}|f|^{2} \frac{d z d \bar{z}}{2 i}
$$

for any $f \in W_{2}^{1}\left(B_{\varepsilon}\right)$.

According to this lemma there exists $\gamma_{i}$ such that

$$
\int_{\Gamma_{i}} Q(\omega, \omega) \geq \gamma_{i} t \int_{\Gamma_{i}}\langle\omega \mid \omega\rangle
$$

for all $\omega \in \Lambda_{2}^{+}(M) \cap D(\bar{\Delta}(t V))$. Collecting together (13) and (14) we obtain:

$$
\int_{M}\langle\bar{\Delta}(t V) \omega \mid \omega\rangle \geq \gamma t \int_{M}\langle\omega \mid \omega\rangle
$$

for some $\gamma>0$. Hence all eigenvalues of $\left.\bar{\Delta}(t V)\right|_{\Lambda_{2}^{+}(M)}$ increase proportional to $t$ for $t \rightarrow \infty$.

Now we have to obtain the corresponding fact for $\Lambda_{2}^{-}(M)$. For all $\forall \omega \in \Lambda_{2}^{-}(M)$, $\omega=p d z+q d \bar{z}$ it is easy to put down $\int_{\Gamma_{i}} Q(\omega, \omega)$ :

$$
\begin{aligned}
\int_{\Gamma_{i}} Q(\omega, \omega)= & 2 \int\left\{|\partial p|^{2}+|\partial q|^{2}+t^{2}|\hat{z}|^{2}\left|h_{1}(z)\right|^{2}\left(|q|^{2}+|p|^{2}\right)\right. \\
& \left.+t p \bar{q} \overline{h_{2}(z)}+t q \bar{p} h_{2}(z)\right\} \frac{d z d \bar{z}}{2 i}
\end{aligned}
$$

where we use the following notations:

$$
h_{1}(z)=\frac{c_{i}}{2}+d_{i}(\hat{z}), \quad h_{2}(z)=\frac{c_{i}}{2}+d_{i}(\hat{z})+\hat{z} \frac{\partial}{\partial z} d_{i}(\hat{z}) .
$$

The two last terms can be rewritten:

$$
j_{1}=t p \bar{q} \overline{h_{2}(z)}+t q \bar{p} h_{2}(z)=t \delta(p \bar{q}+q \bar{p})+2 t \operatorname{Re}\left(\bar{p} q\left(h_{2}(z)-\delta\right)\right) .
$$

Here we introduce real positive parameter $\delta$, which can be defined below.

It is not difficult to obtain the estimate for $j_{1}$ :

$$
j_{1} \geq t \delta(p \bar{q}+q \bar{p})-t\left(|p|^{2}+|q|^{2}\right)\left|h_{2}(z)-\delta\right| .
$$

The term in (15), which is proportional to $t^{2}$ can be rewritten as:

$$
\begin{aligned}
j_{2} & \equiv t^{2}|\hat{z}|^{2}\left|h_{1}(z)\right|^{2}\left(|p|^{2}+|q|^{2}\right) \\
& =\left(t^{2}|\hat{z}|^{2} \delta^{2}+t^{2}\left|h(z)_{1}-\delta\right|^{2}+2 t^{2} \delta \operatorname{Re}\left(h_{1}(z)-\delta\right)\right)\left(|p|^{2}+|q|^{2}\right),
\end{aligned}
$$

and with the condition for the parameter $\delta$ :

$$
\left|h_{1}(z)-\delta\right|>4 \delta
$$

can be estimated:

$$
j_{2} \geq\left(t^{2}|\hat{z}|^{2} \delta^{2}+8 t^{2} \delta^{2}\right)\left(|p|^{2}+|q|^{2}\right) \text {. }
$$


If we take into account that there exists $t_{0}>1$, such that the following inequality takes place:

$$
8 t^{2} \delta^{2}>t \max _{\Gamma_{i}}\left|h_{2}(z)\right| \text { for } t>t_{0}
$$

we can infer that

$$
\int_{\Gamma_{i}} Q(\omega, \omega)=2 \int_{\Gamma_{i}}\left\{|\partial p|^{2}+|\partial q|^{2}+t^{2}|\hat{z}|^{2} \delta^{2}\left(|p|^{2}+|q|^{2}\right)+t \delta(p \bar{q}+q \bar{p})\right\} \frac{d z d \bar{z}}{2 i}
$$

for described $t$. Now it is necessary to use Lemma A.2 proved in Appendix A.

Lemma A.2. For the operator $H$ with Neumann boundary conditions where

$$
H=L \otimes I+\left(\begin{array}{cc}
0 & \beta t \\
\beta t & 0
\end{array}\right), \quad L=-\partial \bar{\partial}+\beta^{2} t^{2}|z|^{2},
$$

there exist $\gamma(\varepsilon, \beta), t_{0}$ such that for $t>t_{0}$ the minimax principle numbers can be estimated:

$$
\mu_{n}(H) \geq \gamma(\varepsilon, \beta) t, \quad n \geq 2 .
$$

So $\int_{M} Q(\omega, \omega) \geq \gamma_{2} t \int\langle\omega, \omega\rangle$ for some $\gamma_{2}>0$ and for $\omega$ which are perpendicular to the first $m$ eigenfunction of $\bar{\Delta}(t V)$.

This equality completes this stage of the proof. Now we can infer that there are only slowly increased eigenfunctions in $\Lambda_{2}^{-}(M)$ [due to supersymmetry arguments, they are zero-modes of $\bar{\Delta}(t V)]$ and other eigenfunctions correspond to the eigenvalues which increase as $t$ for $t \rightarrow \infty$.

To complete the proof of this theorem we have to overcome the conditions which we assumed at the beginning of the proof. Following [4] it is possible to show that smooth deformations of the metric in the vicinities of the superpotential critical points lead to equivalence of the norms on $\Lambda_{2}(M)$. Due to this fact all our estimates take place and for the deformed scalar product and yield same result for the Witten index. To prove that our result does not depend on superpotential critical point degeneracy we have to repeat the proof of the corresponding fact in [6] without any changes in formulas.

Corollary 4.4 (Vanishing theorem). The kernel of $Q$ is a subspace of $\Lambda_{2}^{-}(M)$, i.e.,

$$
\operatorname{Ker}\left(A^{*}\right)=0 .
$$

Corollary 4.5. From the note about the metric deformation at the end the proof we can infer that in our considerations it is possible to use an asymptotically euclidean at infinity metric instead of an euclidean at infinity one.

\section{Concluding Remarks}

In this section we would like to underline two details which we did not discuss enough in the main part of this paper.

The first remark concerns the necessity of including the euclidean at infinity metric. Indeed, due to the identities as

$$
\frac{1}{\pi} \frac{\partial}{\bar{\partial} z} \frac{1}{z}=\delta(z)
$$


the algebra for supercharges contains $\delta$-function terms which have supports at the poles of the superpotential. So, if we want to keep the algebra of supersymmetric quantum mechanics, such terms should be zero in the sense of $L_{2}$-theory. The euclidean at infinity metric gives us this possibility.

In the final remark we would like to note that the expression for the Witten index can be rewritten in a different form. To see this let us consider $D$ - the divisor of the meromorphic differential $\partial f$. It is well-known [17] that

$$
\operatorname{deg} D=2 g-2=-\chi\left(M_{0}\right)
$$

where we use the notation $g$ for the genus of Riemann surface and $\chi\left(M_{0}\right)$ is Euler characteristic of compact Riemann surface $M_{0}$. Then the number of zeros of the form $\partial f$ can be expressed as a sum of the Euler characteristic of the Riemann surface with sign minus and the number of poles of the differential of the meromorphic superpotential and

$$
\Delta_{W} H=\chi\left(M_{0}\right)+\operatorname{deg} \tilde{D}
$$

where $\tilde{D}$ is a divisor of poles of the differential of the meromorphic superpotential.

In this form our result is a pure generalization of the results, which were obtained in [5] for the case of $g=0$. This fact demonstrates once more the observation that the Witten index can be generated by either singularities or topological nontrivial situations.

\section{Appendix A}

In this appendix we are going to give a supersymmetrical proof of Lemma A.1 and Lemma A.2, and by the way, to describe the supersymmetric Hamiltonian on the segment $[-\varepsilon, \varepsilon]$.

We consider the supercharge $Q$ with superpotential $\chi$ such that $\chi \in C^{1}([-\varepsilon, \varepsilon])$, $\chi(-\varepsilon) \neq 0$ and $\chi(\varepsilon) \neq 0$ :

$$
Q=\frac{1}{2}\left(\begin{array}{cc}
0 & \left.e^{\chi} i \partial_{x} e^{-\chi}\right|_{D} \\
e^{-\chi} i \partial_{x} e^{\chi} & 0
\end{array}\right)
$$

where the symbol $\left.\right|_{D}$ means that the operator $\left.e^{\chi} i \partial_{x} e^{-\chi}\right|_{D}$ is provided by the boundary conditions: $\psi(-\varepsilon)=\psi(\varepsilon)=0$.

Corresponding supersymmetric Hamiltonian has the form:

$$
H=\left(\begin{array}{cc}
H_{0} & 0 \\
0 & H_{1}
\end{array}\right)
$$

where $H_{1}=-\left.\frac{1}{4} e^{-\chi} \partial_{x} e^{2 \chi} \partial_{x} e^{-\chi}\right|_{D}$ and $H_{0}=-\frac{1}{4} e^{\chi} \partial_{x} e^{-2 \chi} \partial_{x} e^{\chi}$ with boundary conditions: $\left.\partial_{x} e^{\chi} \psi\right|_{ \pm \varepsilon}=0$.

For the harmonic oscillator superpotential $\chi=\beta t x^{2}$ for some $\beta, t>0$, the supersymmetric component Hamiltonians have the form:

$$
\begin{gathered}
H_{1}=\left(-\frac{1}{4} \partial_{x}^{2}+(\beta t)^{2} x^{2}+\frac{1}{2} \beta t\right),\left.\quad \psi\right|_{ \pm \varepsilon}=0 \\
H_{0}=\left(-\frac{1}{4} \partial_{x}^{2}+(\beta t)^{2} x^{2}-\frac{1}{2} \beta t\right),\left.\quad\left(\partial_{x}+2 \beta t x\right) \psi\right|_{ \pm \varepsilon}=0 .
\end{gathered}
$$


It is easy to see that $H_{1}$ is a positive self-adjoint operator and $H_{0}$ is a nonnegative self-adjoint one. Indeed, $\sigma\left(H_{0}\right)$ - spectrum of $H_{0}$ - contains zero eigenvalue with eigenfunction

$$
\psi_{0}=\operatorname{const} e^{-\beta t x^{2}},
$$

and $\sigma\left(H_{1}\right)=\sigma\left(H_{0}\right) \backslash\{E=0\}$ due to the supersymmetry.

Now let us return to Lemma a.1:

Lemma A.1. There exists $\gamma(\varepsilon, \beta)>0$ and $t_{0}>0$ such that for $t>t_{0}$,

$$
\int_{B_{\varepsilon}}\left\{\left|\frac{\partial f}{\partial z}\right|^{2}+t^{2} \beta^{2}|z|^{2}|f|^{2}\right\} \frac{d z d \bar{z}}{2 i} \geq \gamma(\varepsilon, \beta) t \int_{B_{\varepsilon}}|f|^{2} \frac{d z d \bar{z}}{2 i}
$$

for $\forall f \in W_{2}^{1}\left(B_{\varepsilon}\right)$.

Proof. First of all we note that it is enough to prove this inequality for the square $S=\left[-\frac{\varepsilon}{\sqrt{2}}, \frac{\varepsilon}{\sqrt{2}}\right] \times\left[-\frac{\varepsilon}{\sqrt{2}}, \frac{\varepsilon}{\sqrt{2}}\right]$, because

$$
\begin{aligned}
\int_{B_{\varepsilon}}\left\{\left|\frac{\partial f}{\partial z}\right|^{2}+t^{2} \beta^{2}|z|^{2}|f|^{2}\right\} \frac{d z d \bar{z}}{2 i} \geq & \int_{S}\left\{\left|\frac{\partial f}{\partial z}\right|^{2}+t^{2} \beta^{2}|z|^{2}|f|^{2}\right\} \frac{d z d \bar{z}}{2 i} \\
& +t^{2} \beta^{2} \frac{\varepsilon^{2}}{2} \int_{B_{\varepsilon} \backslash S}|f|^{2} \frac{d z d \bar{z}}{2 i}
\end{aligned}
$$

and if we will prove this lemma for $S$ instead of $B_{\varepsilon}$ for some $\gamma_{S}(\varepsilon, \beta)$, then Lemma A.1 will be proved for $\gamma(\varepsilon, \beta)=\gamma_{S}(\varepsilon, \beta)$ and $t_{0}=\frac{2 \gamma_{S}(\varepsilon, \beta)}{(\beta \varepsilon)^{2}}$. So it is enough to give the proof of the 1-dimension analog of Lemma A.1: There exists $\gamma(\varepsilon, \beta)>0$ such that

$$
a(f, f) \equiv \int_{-\varepsilon}^{\varepsilon}\left\{\frac{1}{4}\left|\partial_{x} f\right|^{2}+t^{2} \beta^{2}|z|^{2}|f|^{2}\right\} d x \geq \gamma(\varepsilon, \beta) t \int_{-\varepsilon}^{\varepsilon}|f|^{2} d x
$$

for $\forall f \in W_{2}^{1}([-\varepsilon, \varepsilon])$.

In order to obtain this inequality let us extract from the l.h.s. the sesquilinear form $a_{0}(\cdot, \cdot)$ corresponding to the operator $H_{0}$ :

$$
\begin{aligned}
a(f, f)= & \int_{-\varepsilon}^{\varepsilon}\left(\frac{1}{4}\left|\partial_{x} f\right|^{2}+\left(t^{2} \beta^{2}|z|^{2}-\frac{1}{2} \beta t\right)|f|^{2}\right\} d x \\
& +\frac{1}{2} \beta t \int_{-\varepsilon}^{\varepsilon}|f|^{2} d x \pm 2 \beta t x|f|_{ \pm \varepsilon}^{2} \\
= & a_{0}(f, f)+\frac{1}{2} \beta t \int_{-\varepsilon}^{\varepsilon}|f|^{2} d x+2 \beta t \varepsilon\left(|f(-\varepsilon)|^{2}-|f(\varepsilon)|^{2}\right)
\end{aligned}
$$


From the above consideration the spectrum of the operator $H_{0}$ is nonnegative and $a_{0}(f, f) \geq 0$ for $f \in W_{2}^{1}([-\varepsilon, \varepsilon])$. The sesquilinear form $a(f, f)$ corresponds to the operator

$$
H=-\frac{1}{4} \partial_{x}^{2}+t^{2} \beta^{2} x^{2} \text { with conditions: }\left.\partial_{x} \psi\right|_{ \pm \varepsilon}=0
$$

This form achieves the lower value on the eigenfunction $\psi_{0}$ of $H$ which is even or odd function. On this function the last term in (19) vanishes and

$$
\frac{a(f, f)}{(f, f)} \geq \frac{a\left(\psi_{0}, \psi_{0}\right)}{\left(\psi_{0}, \psi_{0}\right)} \geq \frac{\beta t}{2}
$$

These inequalities prove Lemma A.1 for $\gamma(\varepsilon, \beta)=\beta$ and $t_{0}=\frac{2}{\beta \varepsilon^{2}}$.

Lemma A.2. For the operator $H$ with Neumann boundary conditions on $B_{\varepsilon}$ where

$$
H=L \otimes I+\left(\begin{array}{cc}
0 & \beta t \\
\beta t & 0
\end{array}\right), \quad L=-\partial \bar{\partial}+\beta^{2} t^{2}|z|^{2}
$$

there exist $\gamma(e, \beta), t_{0}$ such that for $t>t_{0}$, the minimax principle numbers can be estimated:

$$
\mu_{n}(H) \geq \gamma(\varepsilon, \beta) t, \quad n \geq 2 .
$$

Proof. It is clear that using arguments similar to Lemma A.1 we return to the consideration of $S=\left[\frac{-\varepsilon}{\sqrt{2}}, \frac{\varepsilon}{\sqrt{2}}\right] \times\left[\frac{-\varepsilon}{\sqrt{2}}, \frac{\varepsilon}{\sqrt{2}}\right]$.

Minimax principle numbers $\mu_{n}(H) \geq \mu_{2}(H)$ and achieve the values on the eigenfunctions of the operator $H$. We have to consider the equations:

$$
\left(L-\mu_{n}(H)\right) f=\beta t g, \quad\left(L-\mu_{n}(H)\right) g=\beta t f
$$

with Neumann boundary conditions for $f, g \in W_{2}^{1}(s)$, and after the iteration

$$
\left(L-\mu_{n}(H)\right)^{2} f=\beta^{2} t^{2} f .
$$

Using Lemma A.1 we have

$$
\mu_{i}(L) \geq \beta t+2 \mu_{i}\left(H_{0}\right) \text { and } \mu_{1}\left(H_{0}\right)=0
$$

So we cannot be sure that $\mu_{1}(H) \geq \gamma t$ for some $\gamma>0$, but we can definitely say that

$$
\mu_{2}(H) \geq \beta t
$$

This note finishes the proof of Lemma A.2 for $\gamma(\varepsilon, \beta)=\beta$ and $t_{0}=\frac{2}{\beta \varepsilon^{2}}$. 


\section{References}

1. Golfand, Y A., Lichtman, E.P.: Sov. Phys. JETP Lett. 13, 452-455 (1971);

Volkov, D.V , Akulov, V.P : Sov. Phys. JETP Lett. 16, 621-623 (1972);

Wess, J, Zumino, B.: Nucl. Phys. B 70, N1, 39-65 (1974)

2 Witten, E : Constraints on supersymmetry breaking. Nucl. Phys. B 202, 253-316 (1982)

3 Alvarez-Gaumé, L.: Supersymmetry and the Atiyah-Singer index theorem. Commun. Math. Phys. 90, 161-173 (1983)

4. Borisov, N.V., Muller, W., Schrader, R.: Relative index theorems and supersymmetric scattering theory. Commun. Math Phys. 114, 475-513 (1988)

5 Jaffe, A., Lesniewski, A., Lewenstein, M.: Ground state structure in supersymmetric quantum mechanics Ann Phys. 178, 313-329 (1987)

6. Jaffe, A, Lesniewski, A.: Supersymmetric field theory and infinite dimensional analysis. Proceeding of the 1987 Cargèse Summer School, 't Hooft, G et al. (eds )

7. Arai, A.: J. Math. Phys 30, 1164 (1989)

Arai, A.: J Math. Phys. 30, 2973 (1989)

Mathur, M.: Ann. Phys. 204, 233 (1990)

8 Arai, A, Ogurisu, O.: Meromorphic $N=2$ Wess-Zumino supersymmetric quantum mechanics J. Math Phys 32, 2427-2434 (1991)

9. Klimek, V., Lesniewski, A.: Local rings of singularities and $N=2$ supersymmetric quantum mechanics Commun. Math. Phys. 136, 327-344 (1991)

10 Cecotti, S., Girardello, L, Pasquinucci, A : Singularity theory and $N=2$ supersymmetry. Int J Mod. Phys. A 6, 2427-2477 (1991)

11. Strichartz, R.S : Analysis of the Laplacian on the complete Riemannian manifold. J Funct Anal. 52, 48-79 (1983)

12. Grauert, H, Remmert, R: Theorie der Steinschen Räume. Berlin, Heidelberg, New York: Springer 1977

13. Worner, F W.: Foundations of differentiable manifolds and Lie groups. Berlin, Heidelberg, New York: Springer 1983

14. Kato, $\mathrm{T}:$ Perturbation theory for the linear operators. Berlin, Heidelberg, New York: Springer 1984

15 Witten, E.: Supersymmetry and Morse theory. J Diff. Geom. 17, 661-692 (1982)

16 Reed, M., Simon, B.: Methods of modern mathematical physics. V. 1-4, New York: Academic Press 1978

17. Springer, J : Introduction to Riemann surfaces. Reading, Massachusetts, USA: Addison-Wesley Publishing (1957)

Communicated by A Jaffe 